\title{
Precursory phenomena associated with large avalanches in the long-range connective sandpile (LRCS) model
}

\author{
Ya-Ting Lee $^{\mathrm{a}}$, Chien-chih Chen ${ }^{\mathrm{a}, *}$, Young-Fo Chang ${ }^{\mathrm{b}}$, Ling-Yun Chiao ${ }^{\mathrm{c}}$ \\ ${ }^{a}$ Graduate Institute of Geophysics, National Central University, Jhongli, 320, Taiwan, ROC \\ ${ }^{\mathrm{b}}$ Institute of Seismology, National Chung-Cheng University, Ming-Hsiung, Chia-Yi, 621, Taiwan, ROC \\ ${ }^{\mathrm{c}}$ Institute of Oceanography, National Taiwan University, Taipei, 106, Taiwan, ROC
}

\section{A R T I C L E I N F O}

\section{Article history:}

Received 16 January 2008

Received in revised form 9 April 2008

Available online 9 May 2008

\section{Keywords:}

Long-range connection

Long-range interaction

Sandpile model

Precusory phenomena

Intermittent criticality

Seismicity

$b$-value

\begin{abstract}
A B S T R A C T
Reduction in $b$-values before a large earthquake is a very popular topic for discussion. This study proposes an alternative sandpile model being able to demonstrate reduction in scaling exponents before large events through adaptable long-range connections. The distant connection between two separated cells was introduced in the sandpile model. We found that our modified long-range connective sandpile (LRCS) system repeatedly approaches and retreats from a critical state. When a large avalanche occurs in the LRCS model, accumulated energy dramatically dissipates and the system simultaneously retreats from criticality. The system quickly approaches the critical state accompanied by the increase in the slopes of the power-law frequency-size distributions of events. Afterwards, and most interestingly, the power-law slope declines before the next large event. The precursory $b$-value reduction before large earthquakes observed from earthquake catalogues closely mimics the evolution in power-law slopes for the frequency-size distributions of events derived in the LRCS models. Our paper, thus, provides a new explanation for declined $b$-values before large earthquakes.
\end{abstract}

(C) 2008 Elsevier B.V. All rights reserved.

\section{Introduction}

Numerically simulating earthquakes is mainly based on conceptual models such as the spring-slider model of Burridge and Knopoff [7], the block structure model of Gabrielov et al. [12], the lattice-solid model of Mora and Place [26], and the sandpile model of Bak et al. [1]. The spring-slider model [7] and the sandpile model [1] are two types of simple cellular automata models among them. Bak et al. [1] originally introduced the self-organized criticality (SOC) concept. The study of the SOC has been mainly based on simulations using sandpile model. Bak et al. showed their sandpile model can reach a critical state without the need to fine-tune the system parameters. Their model has large avalanches randomly in critical state. The sandpile model can drive itself into a statistically stationary state characterized by spatial and temporal correlation functions exhibiting power-law behavior. The model has no characteristic avalanche size. It covers all possible values with power-law distribution. The exponent of the power law between avalanches size and frequency could be characteristic of the self-organized criticality $([1,2,9,27])$.

Seismic activity is caused by stress accumulation in a rock. The rock fractured by stress accumulation releases energy to the surrounding materials. The surrounding material in the critical state that cannot bear the energy increase would set off a chain of events, namely a large earthquake, caused by energy release from neighboring sites. The universal Gutenberg-Richter power-law relation describes the energy distribution of earthquakes. Gutenberg and Richter in 1956

\footnotetext{
* Corresponding author. Tel.: +886 3422715165653 ; fax: +886 34222044.

E-mail address: chencc@ncu.edu.tw (C.-c. Chen).
} 


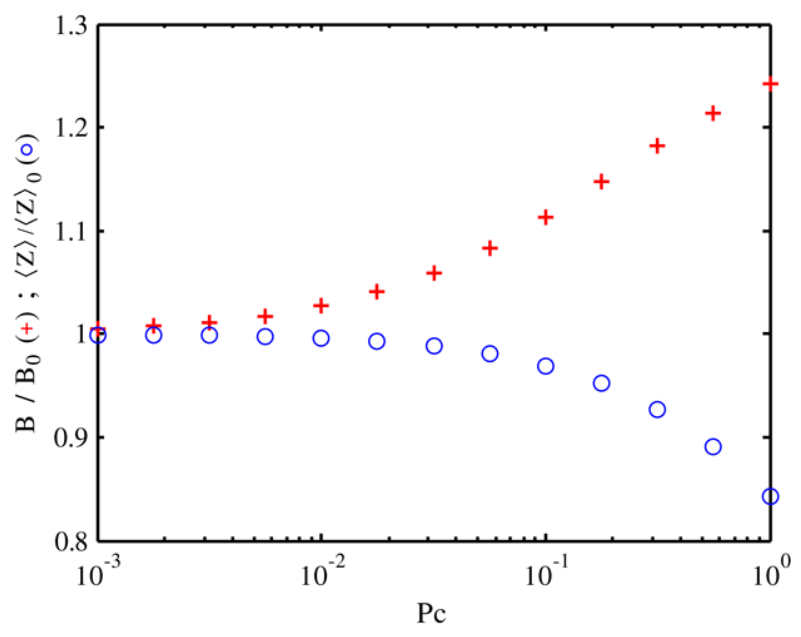

Fig. 1. Scaling exponents (crosses) of the long-range connective sandpile models with various connective probabilities $P_{c}$ gradually increasing from 0 to 1 [10]. All the scaling exponents are normalized to the value of scaling exponent, i.e. $B_{0}=1.06$, of the nearest neighbor sandpile with $P_{c}=0$. Also shown in the plot is the average number of total grains staying on the grid (circles) for each $P_{c}$. Again, the total grain number is normalized to the number for the case of $P_{c}=0$, which is averagely $\langle Z\rangle_{0}=5244$.

noted earthquake frequency where energy larger than $\sim 10^{1.5 M+16.1}$ dyne- $\mathrm{cm}$ is released, following a simple power law, $\log N_{\geq M}=a-b M$. The scaling exponent in the power-law Gutenberg-Richter relation is very well known as the $b$ value. The literature often discusses variation in the $b$-value and considers it a monitoring index related to forthcoming large earthquakes ([31,34,36,38,15,14,19,40,18,16,4,28,41,20,39]). Laboratory experiment results modeling natural faults also clearly show a lower scaling exponent during the foreshock period than during background periods ([37]). Several researchers have reported $b$-value reductions before large earthquakes $([33,21,23,32])$.

Our present paper presents an alternative model reminiscent of real seismic activity, based on the original sandpile model ([1]). The modified sandpile model long-term averagely also has characteristics of power-law frequency-size distribution. The current work proposes an alternative sandpile model variant with random internal connections to demonstrate intermittent criticality and reductions in the scaling exponents before large avalanches.

\section{Long-range connective sandpile models}

We currently build a sandpile model by a very simple set of rules similar to the original Bak-Tang-Wiesenfeld-type sandpile model [1]. For a square grid of $L$ by $L$ cells, we randomly throw sands, one at a time, onto the grid. In the original sandpile model, once the total amount of accumulated sands within a single cell reaches the threshold amount of 4 , they are redistributed to four adjacent cells (the nearest neighbors) or lost off the edge of the grid. All cells receiving grains from their neighbors are checked, and redistribution continued further away if any of them reaches over the threshold. Redistribution for each throw of new grains proceeds until none of the meshes receiving new grains exceeds the threshold. The total amount of cells involved in the redistribution process initiated by a single throw is defined as the size of the event. Note that the total amount of grains retained within the grid increases linearly in the beginning, transient thousands of iterations, and then stays at a quasi-static value with small fluctuations.

The modified rule of random internal connections is implemented by slightly changing the redistribution procedure as the following. When accumulated grains for any particular cell exceed the threshold and redistribution occurs, one of the original nearest neighbor connections faces a connective probability $P_{c}, 0<P_{c}<1$, of redirecting to a randomly chosen, distant cell, replacing the original connection by a randomly chosen mesh that might be faraway from the toppling cell. We may call such a sandpile model version the long-range connective sandpile (LRCS) model. Apparently, when $P_{c}=0$, the LRCS model reduces to the original nearest neighbor sandpile model of Bak et al. [1]. Findings show that the connective probability $P_{c}$ has significant impact on scaling exponents of power-law frequency-size distributions in the LRCS model ([10]). Chen et al. [10] perform a series of simulations each with $10^{6}$ throws of single grain on a square grid with 50 by 50 meshes, starting with the original nearest neighbor sandpile $\left(P_{c}=0\right)$ and then gradually increasing the connective probability $P_{c}$. Each $P_{c}$, robustly emerging has the frequency-size power law and the manifested SOC state. Their investigation finds a systematic steady increase of the scaling exponent along with a notable decrease in total grain amount staying on the grid (Fig. 1) when connective probability $P_{c}$ gradually increases from 0 to 1 .

In recent years, there have been numerous attempts to combine sandpile models with complex networks. Some papers have also studied the role of non-locality in sandpile behavior [25,17]. In addition, there have been attempts to apply sandpile with time-varying exponents and non-trivial connectivity to seismology and earthquake prediction [35]. In this study, more different from previous studies is essentially that we give an additional rule of self-adaptedly tuning $P_{c}$. We 


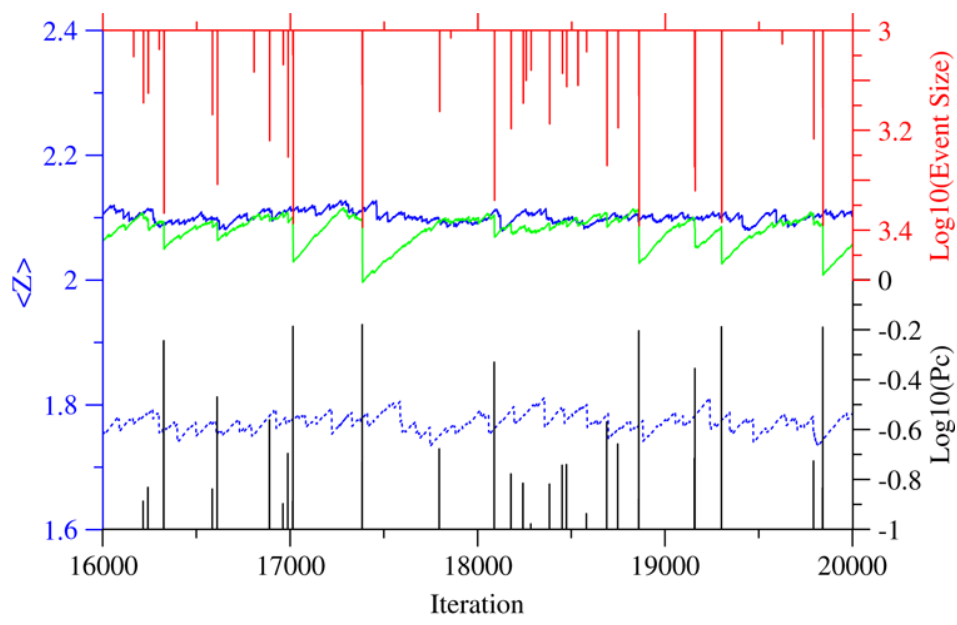

Fig. 2. Temporal evolution of the spatially averaged amount of grains on board $\langle Z\rangle(t)$ for three long-range connective sandpile models with $P_{c}=0$ (blue solid line), $P_{c}=1$ (blue dotted line) and self-adapted $P_{c}$ (green line). Also shown in the plot are the occurrence of avalanches with size $>1000$ (downward red bars) and $P_{c}$ larger than 0.1 (upward black bars) in the corresponding sandpile model with variable $P_{c}$. (For interpretation of the references to colour in this figure legend, the reader is referred to the web version of this article.)

assume that $P_{c}$ strongly depends on topographic change in last generation throwing single grain on the grid. Consider that topographic height of the sandpile at the time step $i$ is $\boldsymbol{Z}_{i}(\mathbf{x})$. At the next time step $i+1$, due to the throw of single grain on the grid, it changes from $Z_{i}(\mathbf{x})$ to $Z_{i+1}(\mathbf{x})$. Therefore, total change in the topographic height of the sandpile is $\Delta Z(i+1)=\sum_{x_{l}}\left|Z_{i+1}\left(x_{l}\right)-Z_{i}\left(x_{l}\right)\right|$. Usually, on a square $L$ by $L$ grid, $\Delta Z$ ranges from 1 , which means no toppling occurs, to $\alpha L^{2}(\alpha \cong 1.25)$. We then simply define $P_{c}(i+1)=\left[\Delta Z(i+1) / \alpha L^{2}\right]^{3}$. The meaning for the coefficient $\alpha$ is basically more like the normalization constant, which makes the $P c$ value of the connective probability range between 0 and 1 . It is very likely that there are many other different ways to define $P_{c}$ for different physical systems. At the end of demonstrating intermittent criticality in the sandpile model, we have found that the exact choice of formulation for $P_{c}$ is not crucial. The system after a large avalanche can thus evoke a higher connective probability $P_{c}$ value. Our motivation is that a more active system will have higher probability to establish the long-range connection due to, for example, dynamic triggering of seismic waves. Intuitively, a larger earthquake generates more radiated energy carried by seismic waves, thus, is more capable of dynamically triggering remote tremors far away the main shock. It has been noted that seismicity increased in Long Valley Caldera following the $M_{w}$ (moment magnitude) $=7.92002$ Denali Fault earthquake, $M_{w}=7.11999$ Hector Mine earthquake and $M_{w}=7.31992$ Landers earthquake. The three main shocks were 3460, 400, and $440 \mathrm{~km}$ from Long Valley, respectively. In these long-range triggering cases, the stress perturbation of seismic waves is the immediate cause of the triggered earthquakes.

The self-adapted long-range connective sandpile models represent a family of intermittent criticality. The concept of intermittent criticality $[30,29,8,24,13]$ is based on the hypothesis that a great earthquake on the fault network cannot occur until regional criticality threshold has been reached and stress is consequently correlated at all length scales up to the size of the region. This great event then destroys the overall regional criticality on its associated network. After a period of relative quiescence following the great event, the dynamical process of the earthquake fault system repeats by reloading energy and rebuilding correlation lengths towards the criticality that leads to the next great event. The behavior of intermittent criticality could be found in Fig. 2, in which large avalanches dramatically cause simultaneous energy reductions. For the LRCS model with self-adapted $P_{c}$ the dynamic variable of $\langle Z\rangle(t)$ (green line) is often punctuated towards a smaller value by a large event while its baseline approximately corresponds to the average of $\langle Z\rangle(t)$ in the case of constant zero $P_{c}$ (blue solid line). The large fluctuation in $\langle Z\rangle(t)$ is an important and interesting feature mimicking intermittent criticality [30,29, $8,24,13]$. Large avalanches are then followed by a period of quiescence and a new approach back toward the critical state. This is similar to the dynamical process of the earthquake fault system which repeats by reloading energy and rebuilding correlation lengths towards criticality and the next great event [29].

\section{Scaling exponent in the size distribution of avalanches}

A numerical sandpile system with $2 \times 10^{4}$ throws of single grain on a square grid of $50 \times 50$ meshes has about 8000 pieces of avalanches. We have calculated the scaling exponent in the frequency-size distribution of avalanche events. Hereafter we call the scaling exponent in the frequency-size distribution of avalanches the $B$-value. Throughout this paper the capital $B$ is exclusively used for denoting the scaling exponent of the power-law frequency-size distribution of avalanches. The sliding window technique was adopted, selecting 250 events of avalanches to each calculation of the $B$-value and then repeatedly shifting 25 events to obtain the next $B$-value calculation. The finite data with 250 events would cause noise in the 


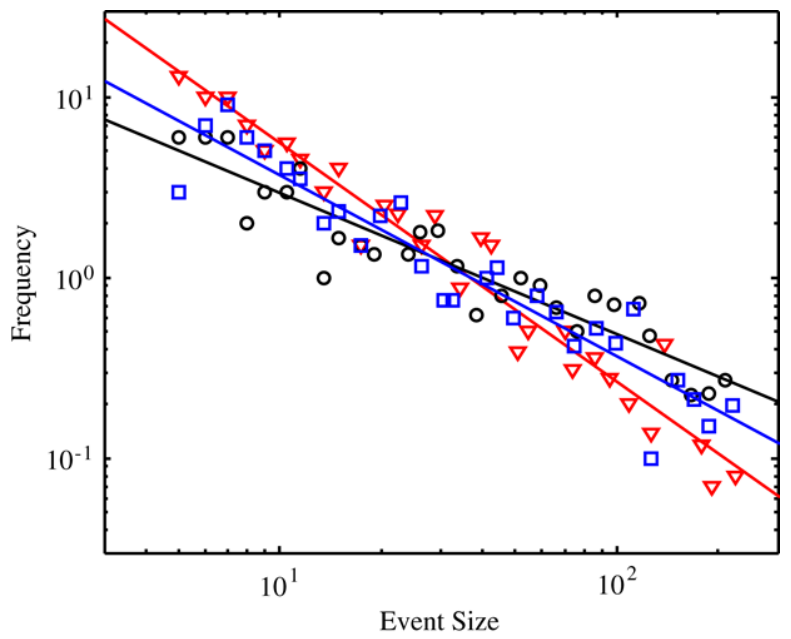

Fig. 3. Power-law frequency-size distributions of avalanches for the self-adapted long-range connective sandpile models. The plot shows three example of distributions with high scaling exponent greater than 1.3 (red inverted triangles), middle scaling exponent of about 1 (blue squares), and low scaling exponent smaller than 0.8 (block circles). Also shown in this plot are three corresponding fitting lines for those distributions. (For interpretation of the references to colour in this figure legend, the reader is referred to the web version of this article.)

statistics, particularly for data with large avalanche sizes. We may apply the data binning technique to extract information from the noisy tail. Data binning reduces the noise for large avalanche sizes and approximates the underlying avalanche size probability well. For details of the data binning technique we refer readers to Christensen and Moloney [11]. We then adopted the standard least-square method to fit the size distribution of avalanches. Fig. 3 displays three examples of high $(>1.3$, red inverted triangles), middle $(\sim 1.0$, blue squares) and low $(<0.8$, black circles) $B$-values. Also shown in this plot are their corresponding fitting lines. Although data points of the distributions seem kind of noisy, $B$-values do change group by group.

Fig. 4(a) shows the $B$-value variation (red line) with different time windows for the LRCS model. The $B$-value, following a large avalanche, usually increases with accumulated energy until the system attains to critical state, and then the $B$-value starts to reduce. It seems that the system moving up to critical state experiences large avalanches. As a demonstration, we plot $10^{4}$ generations in Fig. 4(a), showing a total of seven large avalanches with the sizes more than 2400 . The $B$-value typically reduces prior to those large avalanches and increases after those avalanches. Note that the $B$-values vary from 0.72 to 1.32 for the LRCS model. For comparison, Fig. 4(b) shows results from the original sandpile model with constant $P_{c}=0$, where $\langle Z\rangle(t)$ fluctuates insignificantly at about an average value of 3.6 (blue line). Such a statistically stationary value of $\langle Z\rangle(t)$ is recognized as a manifestation of the SOC state ([3]). Most importantly, in the original BTW-type sandpile, there is no correlation between the $B$-value reductions and large avalanches (red line in Fig. 4(b)). Such observation shows the possibility of detecting precursors of great events in our LRCS model.

Also, for considering the possible finite-size effect in a square grid with 50 by 50 meshes, we have further changed into the grid size $100 \times 100$ (Fig. 5) and taken 500 events within each sliding window for calculating the time-dependent scaling exponent. We also plot $10^{4}$ generations in Fig. 5, showing a total of six large avalanches with the sizes more than 10000 . Fig. 5(a) shows the $B$-value variation with different time windows, varying from 0.8 to 1.6 , for the LRCS model. For comparison Fig. 5(b) shows results obtained from the nearest-neighbor sandpile model, where $\langle Z\rangle(t)$ fluctuates insignificantly around an average value of 3.6. Simply comparing Fig. 4(b) with Fig. 5(b) for the nearest-neighbor sandpile models, most of the obtained scaling exponents for those two grid sizes lie around unity, implying no severe biases due to the smaller size of $50 \times 50$ grid or 250 events per window. Most importantly, those error bars in Fig. 4(a), Fig. 5(a) suggest that the fluctuation in the power-law exponents is really significant for the precursory dynamics in the LRCS model.

\section{Discussion}

A family of popular earthquake physics model is mainly based on cellular automata. The Bak-Tang-Wiesenfeld-type sandpile model [1] is initially a cellular automaton similar to the earthquake fault system, with the SOC character. The sandpile model arrives at criticality by itself, and keeps its critical state. Other researches (e.g. [24,13,29]), on the other hand, suggest that the earthquake fault system is more likely with intermittent criticality. The earthquake fault system is far away from critical state after large events occur. Differentiating between SOC and intermittent critical state of the fault system is important for understanding earthquake physics. Sammis and Smith [30] presented a new class of cellular automata models. They built a large scale heterogeneity system, fractal automaton, and studied the effect of loss factor on system behavior. A large event in those models would be followed by a shadow period of quiescence and then approach back toward the critical state. Castellaro and Mulargia [8] analyzed six different 2-D prototype cellular automata models. Their system approaches 

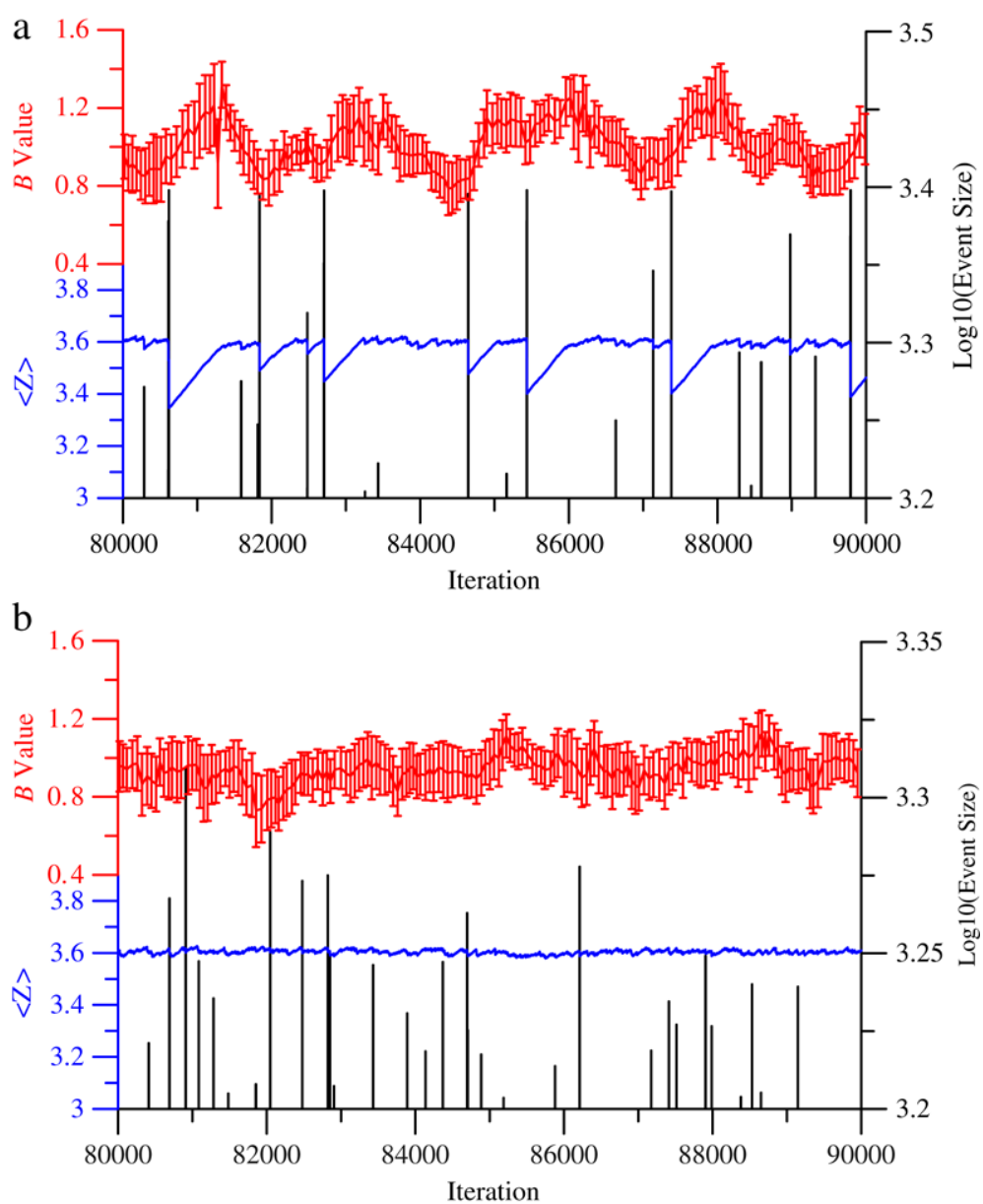

Fig. 4. For a square grid of 50 by 50 cells, blue lines represent the dynamic variable of the average topographic height of the sandpile $\langle Z\rangle(t)$ in (a) the LRCS model with self-adapted $P_{c}$ and (b) the original Bak-Tang-Wiesenfeld-type sandpile model. Red lines are the variation in the scaling exponents. Error bar show the $95 \%$ confidence intervals. The plot also shows avalanche occurrence (black bars) with size $>1500$. (For interpretation of the references to colour in this figure legend, the reader is referred to the web version of this article.)

and retreats from a critical state. Note that all of the models mentioned above - displaying intermittent criticality - have prescribed tuning parameters. Our model with self-adapted $P_{c}$ also displays similar intermittent criticality. However, there is no tuning parameter in our modified LRCS sandpile model.

Moreover, the literature uses many variables for detecting precursory phenomena of a forthcoming great event (e.g., $[24,13,5,6])$. Several authors believe that change in the seismic $b$-value could be a statistically significant precursor. Main [22] studies damage mechanics with long-range interactions, caused by the elastic stress field. Long-range interaction between cracks in their laboratory creep experiments is taken as elastic, implying a positive interaction potential proportional to crack size. This interaction also explains major temporal fluctuation in $b$-value in terms of the underlying physical process of time-varying applied stress and crack growth under constant stain rate. Main [21] shows that $b$-value varies between 0.5 and 1.5 during the earthquake cycle, and that critical failure in a large earthquake should occur at $b=0.5$. They also discuss $b$-value evolution for laboratory creep experiments and seismicity during the Coalinga earthquake near Parkfield California (Fig. 1(d) in Main [21]; Fig. 8 in Main [23]). Their studies suggest that before failure the $b$-value decreases as stress increases. Results from the southern California earthquake catalogue, where seismicity precedes each large main shock is associated with low seismic $b$-values [15]. Suyehiro [33] also presented the examples of foreshocks with low $b$-values for the 1960 Chilean and the 1964 Japanese earthquakes. The $b$-values of their foreshocks $(0.55$ in the Chilean case and 0.35 in the Japanese case) are smaller than the aftershocks (1.33 for Chilean and 0.76 for Japanese) [33]. The other case is the 1989 Loma Prieta earthquake; the events from mid-1988 to 1989 have a $b$-value of 0.58 substantially lower than the value of 1.13, prevailing from 1979 to 1988 [32]. Wiemer et al. [42] on the other hand, present that the $b$-value grows after both the Landers and Hector Mine main shocks.

Results of the present study indicate that the LRCS model also displays $B$-value reduction before large avalanches and growth after large avalanches. The increase of $B$-value is caused by a system far away from critical state with energy deficit. As the LRCS system moves up to critical state, $B$-values start to reduce. The next large avalanche prepares to occur at this time. 

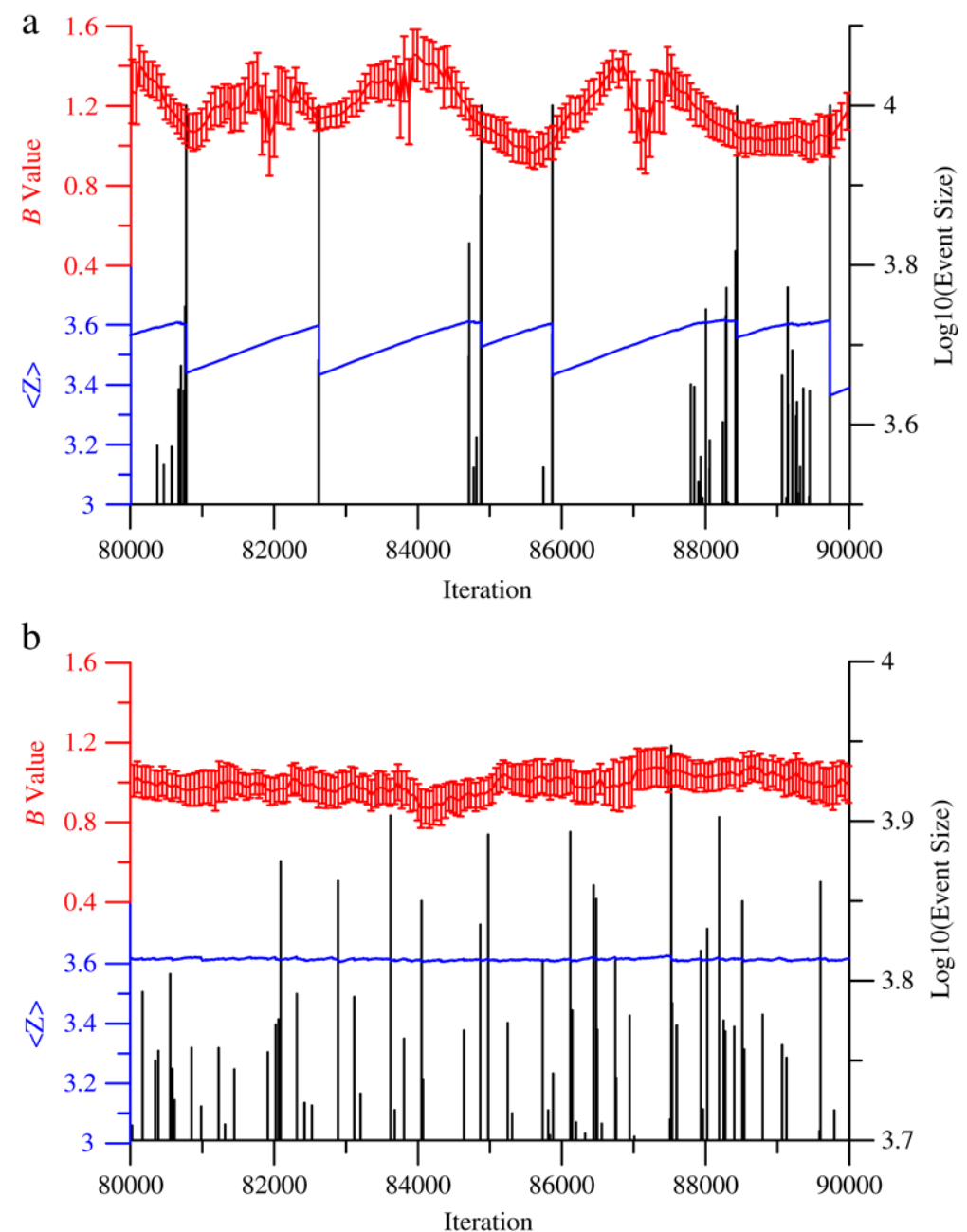

Fig. 5. For a square grid of 100 by 100 cells, blue lines represent the dynamic variable of the average topographic height of the sandpile $\langle Z\rangle(t)$ in $(a)$ the LRCS model with self-adapted $P_{c}$ and (b) the original Bak-Tang-Wiesenfeld-type sandpile model. Red lines are the variation in the scaling exponents. Error bars show the $95 \%$ confidence intervals. The plot also shows avalanche occurrence (black bars) with size $>3000$. (For interpretation of the references to colour in this figure legend, the reader is referred to the web version of this article.)

If the LRCS model is similar to a real earthquake fault system, we can know that as large earthquakes occur system energy reduces quickly, leaving the system far from critical state. Afterward, the system accumulates energy, and approaches critical state again and so on, intermittently. Therefore, low seismic $b$-values may also be importantly associated with precursory quiescence phenomenon.

\section{Conclusion}

This work proposes an alternative sandpile model variant through adaptable long-range connections. Long-range connection probability $\left(P_{c}\right)$ of the LRCS model evolves according to the topographic change induced by the last event. This model demonstrates intermittent criticality. Fig. 2 shows that energy decreases as large events occur. Accordingly the system moves out of critical state and returns back toward the critical state waiting for the next large event. Adaptable long-range connection probability is a strong controlling variable, inducing the system to a state of intermittent criticality.

Another interesting finding is that the variation in the scaling exponent before and after large avalanches has a striking behavior in the LRCS system. When a system has long-range connections, the avalanche dynamics has both local and global characters ([17]). The links to the nearest neighbors, like in a system without long-range connections, give rise to the local events, whereas the long-range connections induce two phenomena, on the one hand by removing grains from the local avalanche and on the other by facilitating an initiation of another local avalanche in the other end of the long range link. The grain removal causes the local avalanches to relax and thus halt quicker, i.e. damping down the avalanche size, while the long-range jumps tend to increase the avalanche activity, i.e. nucleating new local avalanches. It thus could be expected that, 
before a large event, the system probably shuts off most of the long-range connections and stops the relaxation process of long-range grain removals, meaning the activity of small local avalanches decreases and consequently the values of scaling exponent declines.

Our study suggests that seismic $b$-value reduction might be caused by changes in criticality and that large earthquakes would possibly occur as the system moves up to critical state. This study concludes that long-range connection should be integrated into the numerical simulation process of seismicity. Moreover, we can use the LRCS model to test other variables for detecting precursory phenomena of a forthcoming great event in a real earthquake fault system and explain that. This work also tries to improve the seismicity model to better represent real earthquake activity.

\section{Acknowledgements}

CCC is grateful for research support from both the National Science Council (ROC) and the Institute of Geophysics (NCU, ROC). LYC is supported by the National Science Council of the Republic of China under grant NSC 96-2611-M-002-001. Thanks are also extended to the two anonymous reviewers for improving the manuscript.

\section{References}

[1] P. Bak, C. Tang, K. Wiesenfeld, Self-organized criticality: An explanation of 1/f noise, Phys. Rev. Lett. 59 (1987) 381-384.

[2] P. Bak, C. Tang, K. Wiesenfeld, Self-organized criticality, Phys. Rev. A 38 (1988) 364-374.

[3] P. Bak, C. Tang, Earthquakes as a self-organized critical phenomenon, J. Geophys. Res. 94 (1989) 15635-15637.

[4] D.J. Barton, G.R. Foulger, J.R. Henderson, B.R. Julian, Frequency-magnitude statistics and spatial correlation dimensions of earthquakes at Long Valley caldera, California, Geophys. J. Int. 138 (1999) 563-570.

[5] Y. Ben-Zion, M. Eneva, Y. Liu, Large earthquake cycles and intermittent criticality on heterogeneous faults due to evolving stress and seismicity, J. Geophys. Res. 108 (B6) (2003) 2307, doi:10.1029/2002JB002121.

[6] D.D. Bowman, C.G. Sammis, Intermittent criticality and the Gutenberg-Richter distribution, Pure appl. geophys. 161 (2004) 1945-1956

[7] R. Burridge, L. Knopoff, Model and theoretical seismicity, Bull. Seismol. Soc. Amer. 57 (1967) 341-371.

[8] S. Castellaro, F. Mulargia, What criticality in cellular automata models of earthquakes? Geophys. J. Int. 150 (2002) 483-493.

[9] K Chen, P. Bak, Self-organized criticality in a crack-propagation model of earthquakes, Phys. Rev. A 43 (1991) 625-630.

[10] C.C. Chen, L.Y. Chiao, Y.T. Lee, H.W. Cheng, Y.M. Wu, Long-range connective sandpile models and its implication to seismicity evolution, Tectonophysics (2008), doi:10.1016/j.tecto.2008.04.004.

[11] K. Christensen, N.R. Moloney, Complexity and Criticality, Imperial College Press London Inc., London, 2005.

[12] A.M. Gabrielov, T.A. Levshina, I.M. Rotwain, Block model of earthquake sequence, Phys. Earth. Planet. Inter. 61 (1990) 18-28.

[13] C. Goltz, M. Bose, Configurational entropy of critical earthquake populations, Geophys. Res. Lett. 29 (51)(2002) 1-4.

[14] Z. Guo, Y. Ogata, Correlation between characteristic parameters of aftershock distributions in time, space, and magnitude, Geophys. Res. Lett. 22 (1995) 993-996.

[15] J. Henderson, I.G. Main, R.G. Pearce, M. Takeya, Seismicity in north-eastern Brazil - fractal clustering and the evolution of the $b$-value, Geophys. J. Int. 116 (1994) 217-226.

[16] J.R. Henderson, D.J. Barton, G.R. Foulger, Fractal clustering of induced seismicity in the Geysers geothermal area, California, Geophys. J. Int. 139 (1999) 317-324.

[17] J. Lahtinen, J. Kertesz, K. Kaski, Sandpiles on Watts-Strogatz type small-worlds, Physica A 349 (2005) 535-547.

[18] V. Lapenna, M. Macchiato, L. Telesca, $1 / f$ fluctuations and self-similarity in earthquake dynamics - observational evidences in southern Italy, Phys. Earth. Planet. Inter. 106 (1998) 115-127.

[19] D. Legrand, A. Cisternas, L. Dorbath, Multifractal analysis of the 1992 Erzincan aftershock sequence, Geophys. Res. Lett. 23 (9) (1996) 933-936.

[20] P. Mandal, A.O. Mabawonku, V.P. Dimri, Self-organized fractal seismicity of reservoir triggered earthquakes in the Koyna-Warna seismic zone, western India, Pure Appl. Geophys. 162 (2005) 73-90.

[21] I.G Main, P.G. Meredith, C. Jones, A reinterpretation of the precursory seismic $b$-value anomaly from fracture mechanics, Geophys. J. 96 (1989) 131-138.

[22] I.G. Main, Damage mechanics with long-range interactions: Correlation between the seismic $b$-value and the two point correlation dimension, Geophys. J. Int. 111 (1992) 531-541.

[23] I.G. Main, J.R. Henderson, P.G. Meredith, P.R. Sammonds, Self-organised criticality and fluid-rock interactions in the brittle field, Pure Appl. Geophys. 142 (1994) 529-543.

[24] I.G. Main, F.H. Al-Kindy, Entropy, energy, and proximity to criticality in global earthquake populations, Geophys. Res. Lett. 29 (7) (2002) 1121. doi:10.1029/2001GL014078.

[25] S.S. Manna, Two-state model of self-organized criticality, J. Phys. A 24 (1991) 363-369.

[26] P. Mora, D. Place, Simulation of the frictional stick-slip instability, Pure Appl. Geophys. 143 (1994) 61-87.

[27] Z. Olami, H.J.S. Feder, K. Christensen, Self-organized criticality in a continuous, nonconservative cellular automaton modeling earthquakes, Phys. Rev. Lett. 68 (1992) 1244-1247.

[28] A.O. Oncel, T. Wilson, Correlation of seismotectonic variables and GPS strain - measurements in western Turkey, J. Geophys. Res. 109 (B11) (2004) B11306.

[29] J.B. Rundle, W. Klein, D.L. Turcotte, B.D. Malamud, Precursory seismic activation and critical-point phenomena, Pure Appl. Geophys. 157 (2000) 2165-2182.

[30] C.G. Sammis, S.W. Smith, Seismic cycles and the evolution of stress correlation in cellular automaton models of finite fault networks, Pure Appl. Geophys. 155 (1999) 307-334.

[31] W.D. Smith, Evidence of precursory changes in the frequency-magnitude $b$ value, Geophys. J. R. Astron. Soc. 86 (1986) 815-838.

[32] W.D. Smith, Resolution and significance assessment of precursory changes in mean earthquake magnitudes, Geophy. J. Int. 135 (1998) 515-522.

[33] S. Suyehiro, Difference between aftershocks and foreshocks in the relationship of magnitude to frequency of occurerence for the great chilean earthquake of 1960, Bull. Seism. Soc. Am 56 (1966) 185-200.

[34] T.I. Urbancic, C.I. Trifu, J.M. Long, R.P. Young, Space-time correlations of $b$ values with stress release, Pure Appl. Geophys. 139 (1992) $449-462$.

[35] V. Uritsky, N. Smirnova, V. Troyan, F. Vallianatos, Critical dynamics of fractal fault systems and its role in the generation of pre-seismic electromagnetic emissions, Phys. Chem. Earth 29 (2004) 473-480

[36] D. Weatherley, P. Mora, M.F. Xia, Long-range automaton models of earthquakes: Power-law accelerations, correlation evolution, and mode-switching, Pure Appl. Geophys. 159 (2002) 2469-2490.

[37] J. Weeks, D. Lockner, J. Byerlee, Change in $b$-value during movement on cut surfaces in granite, Bull. Seismol. Soc. Amer. 68 (1978) $333-341$.

[38] S. Wiemer, M. Wyss, Seismic quiescence before the landers $(M=7.5)$ and big bear $(M=6.5) 1992$ earthquakes, Bull. Seismol. Soc. Amer. 84 (1994) 900-916.

[39] Y.M. Wu, L.Y. Chiao, Seismic quiescence before the 1999 Chi-Chi, Taiwan Mw7.6 earthquake, Bull. Seismol. Soc. Amer. 96 (2006) 321-327. 
[40] M. Wyss, Nomination of precursory seismic quiescence as a significant 307 precursor, Pure Appl. Geophys. 149 (1997) 79-114.

[41] M. Wyss, C.G. Sammis, R.M. Nadeau, S. Wiemer, Fractal dimension and $b$-value on creeping and locked patches of the San-Andreas fault near Parkfield, California, Bull. Seismol. Soc. Amer. 94 (2004) 410-421.

[42] Stefan Wiemer, Matt Gerstenberger, Egill Hauksson, Properties of the aftershock sequence of the 1999 Mw 7.1 Hector Mine earthquake: Implications for aftershock hazard, Bull. Seismol. Soc. Amer. 92 (2002) 1227-1240. 